\title{
Application of BIM in the protection of historical buildings based on
}

\section{Riegel historic value theory}

\section{Li Yan ${ }^{12}$ Song Zhaoxiang ${ }^{1}$ Dong Kai ${ }^{3}$}

(1, College of Civil Engineering and Architecture, Hainan University, Hainan Haikou 570228, China

2. School of Management and Economics, Tianjin University, Tianjin 300072, China, Institute of International Tourism Island, Hainan University, Hainan Haikou 570228, China

3. School of Economics and Management, Tianjin Urban Construction University, Tianjin 300384, China)

Key words: Riegel historic value theory, Protection of historical buildings ,Building information model

Abstract: Historical building protection has its own features and difficulties. BIM technology, brought by the development of computer technology, make it possible to solve the problem of historical building protection. Based on Riegl value theory, this paper puts forward that different measures should be taken according to different value. The application of BIM in historical building protection was researched from different angle of the value protection to provide the basis for historical building protection and further application of BIM technology in historical building protection.

Fund Project: Natural Science Fund Project in 2013 the Hainan Province "the design and establishment of holographic database Based on time series of historical buildings in Hainan Province", project number 713182

\section{The Value Of Historical Building Protection}

\section{Protection of Historical Buildings and The Historical Architecture}

The totally Historical building we talking about is having certain fixed number of year and protection value historical building and modern architecture which including all levels of cultural relic buildings and cultural relics. Historical and cultural city town village protection regulations, issued in 2008, differentiate between the historical buildings and cultural relics in concept and make a new definition for historic buildings. It is definition of regulation that determined by way of county people's governments of cities, have certain protection value can reflect historical landscape and local features, not released for the cultural relics protection unit, also did not register as immobile cultural relic buildings Structures [1]. Besides, its protection level is higher than general construction and lower than various historical building.

\section{Riegel Value of Historic Buildings}

Riegel, a man who has been hailed as a heritage conservation the Austrian art historian of the prophet, not only from the historical building to analyze the value of itself, but also from the feelings of people to think about the value of historical architecture brings to the modern people, put forward the famous theory of value of historic buildings [2]. As shown in Figure 1.In this paper, the classification of the value of historic buildings to explore the protection of historical buildings from Riegel value theory. Different classification methods, demonstrated the value of different subject 
their different values and methodology, also affects the concept and working mechanism of protection work. [3]

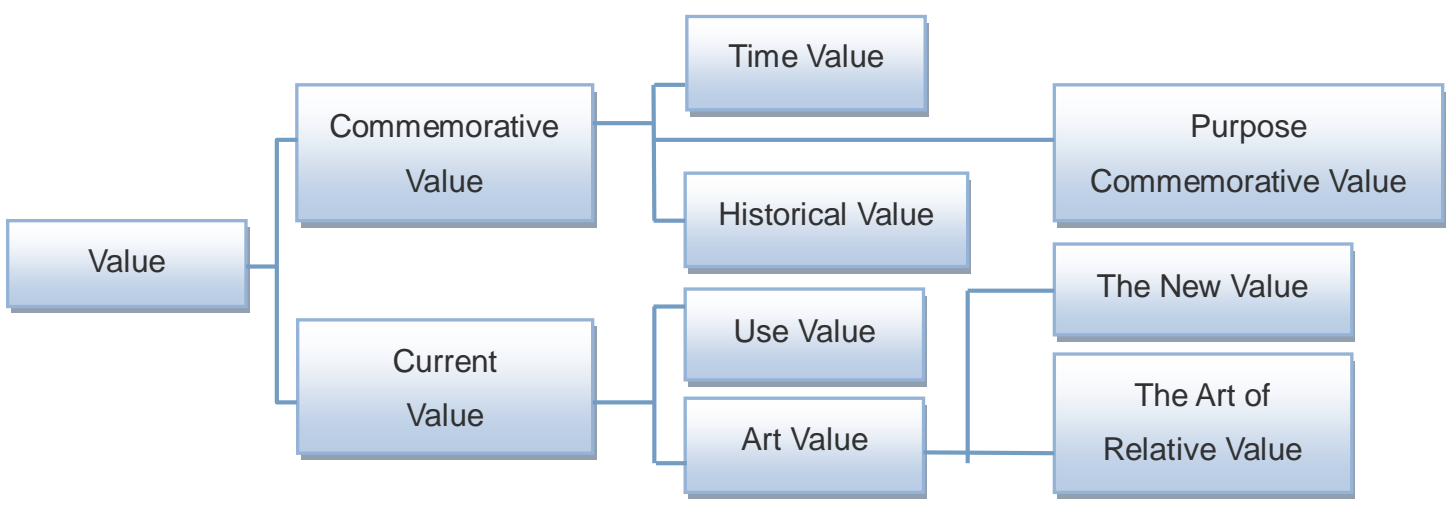

Figure 1, The Classification Value of Historic Buildings by Riegel

"Time Value" refers to the time precipitation in the historical buildings ,so as to make the historic buildings have the ability to infect humans and cause people reverence, worship and value.Architecture is a carrier of the time, people can feel the time in the changes of the building, this perception is not need professional knowledge and specialized training, as, in the point of view: "Time Value to transcend the differences in education and art understanding, because even the simplest mind farmers also can find out the difference between an old clock tower and a new tower."

"Historical Value" means that the historical building, as the crystallization of human intellectual culture, embodies the characteristics of human activities in a historical stage of development, thus contains the commemorative significance. Derived from the historical building material evidences can be used as a historical property, "Historical Value" is the most important differences between historical architecture and general architecture.

"Purpose Commemorative Value", mainly for a memorable historical building, also means monuments we said today. Builders hope future generations may remember a certain historical events or deeds for long in the heart by the construction of a monument.

"Use Value", generally speaking, signifies the structure of the actual function. Usually people make it satisfy the requirement of function to maintain or change the structure of the present situation now. If a building without certain landscape effect, losing the basic function as building at the same time, merely exist for exist.

"Art Value" is a kind of aesthetic value. Its pursuit of art form influenced by modern viewer aesthetic view, it also affected by the time that the aesthetic orientation of the people according to certain period to determine how to protect or repair. "Art Value" contains "New Value" and "The Art of Relative Value". "New Value" content to "The Art of Relative Value" of relative difference is that: the "New Value" is a kind of new art, a work with new state, through visual perception to express themselves clearly. It directs people not only from the angle of human creation to appreciate the predecessors' work, but also from the aspects of its special shape and color. "The Art of Relative Value" influenced by the social main culture interest, may be only a person who have a certain aesthetic foundation can realize. 


\section{BIM technology characteristic and historical building protection}

\section{Integrated Multidimensional Information Transmission}

First of all, BIM has the information sharing platform, supporting for a variety of ways to express and transmit data information. BIM software can not only provide visualization of 3D data, also support form the output of the $2 \mathrm{D}$ drawings, according to the requirements of rendering and imitating animation included in this simulation results expression animation, etc. Under the background of rapid information age, information model can support by network transmission of the EML(Extensible Markup Language) [5]. Secondly, the use of the new model of BIM development historical building protection considers protection and full use of the raw data. BIM links to other relevant original digital data together. Finally, the historical architecture covers a wide variety of information diverse formats and BIM in combination with cloud storage provides vast amounts of data storage space so that all aspects of the data and information to historic buildings together.

\section{Dynamic Coordination Architecture Protection}

Construction project life cycle Management (Building Lifecycle Management, BLM) is the best mode of the current construction project management. BLM, based on BIM technology, create digital management to share information and coordinate the work of the late from design and construction to maintenance, thus reducing the building because of improper coordination in the entire life cycle of invalid behavior and all kinds of risks.

Historical building diversified values, each protecting behavior may affect a variety of value; Historical building protection of diversified participants, a link to a small mistake can lead to serious consequences. Therefore, historical building is reliable than ordinary buildings more emphasis on collaboration and sharing information resources. BIM transform historical building information into digital repository.

\section{Visibility to Alleviate Value Conflicts}

On the one hand, historical building protection level is lower than the historical building protection level, because there is no mandatory article protection. So in the process of urban expansion of economic development in the history of the building tend to be regarded as obstacles to remove. On the other hand, in order to make historic buildings continue to realize the "Use Value", people will to modify historic buildings, but reform is essentially the process of alignment "Historic Value" and "Time Value".

Virtualization formation makes historic buildings into 3D visualization documents. It can realize the historical building preservation and to extract information, even can simulate the effect of the users involved. As a witness of the history of historical architecture visualization permanently preserved, even if the historic buildings will be modified due to the economic development or due to the use needs.

\section{BIM technology in different value of the historical building protection in concrete application}

\section{Historical Value __ Virtual Restoration in Urban Structure Adjustment to The Application of Historical Building Protection}

The "Historical Value" of historic buildings is derived from its role as a physical history witness. So the "Historical Value" of historic buildings should strive to maintain the historic significance to the representative of all times to avoid its gradual decay and wear because of the pure time and the power of nature. It should be properly kept about the present situation of building removal decline. 
The further development of the city needs more urban space, but occupied the urban economic centre of historic buildings get against with tall modern buildings, such as marks the era of the development industrial heritage. "Geographic Value" has skyrocketed and the original old building structure using single function or short of the original use functions of contradictions increasingly obvious.

BIM is widely used in the construction industry in recent years. Integrated 3D imaging characteristics of BIM technology in the complicated historical building protection work has more obvious advantages. These techniques are based on using laser to determine the scope and geometric digital photos to provide geometric model and texture, and the final software platform makes virtual environment construction and calculation the model environment [6]. In addition, the point cloud is grid after processing to establish a solid surface, further generate complex surface form. Finally, other related information from point cloud mapping to historic buildings, such as surface texture material information, etc., finally forms the historical building $3 \mathrm{~d}$ document [7], as shown in figure 2.

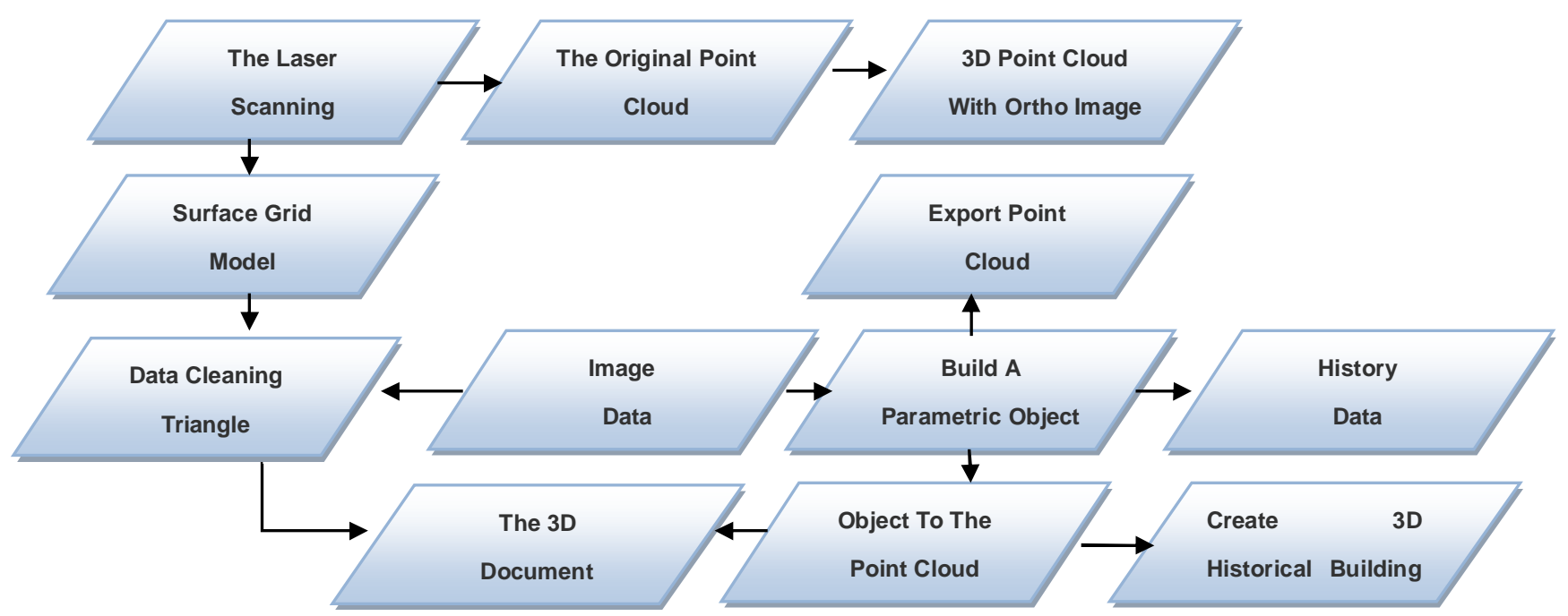

Figure2. Virtual Recovery Process

\section{Artistic value Use value build parameterized component applied in the reconstruction for historical architecture}

The "New Value" of "Art Value" is derived from a new complete building state in historical building. For historical building measures, it should be to remove any traces of old building renovate. The "Art of Relative Value" is derived from the associated to history and modern comprehensive modern aesthetics, and it does not emphasize the importance of the new state, but emphasize the value and enjoy with the eye of art in the construction .It requests decision according to the aesthetic view of modern historic buildings that should be reserved to repair or removal.

In the long history of China, ancient buildings form the unique structural system. Numerous categories, the form of heavy and complicated, the structure of kit kat style and elegant art deco style, they are totally called the most of the world. Because this kind of historical building forms in complicated structure, it is hardly to use traditional software repairing reconstruction or reform, and almost worthless.

The traditional Chinese architecture fully reflects the thought of "Small to Large", and its design is usually from the construction details. First, determine components of geometry size and combine them into architecture. The whole process is in strict accordance with the modular system [8]. This 
is in correspondence with BIM characteristics and requirements of parametric design, so as to establish the parameterized model of ancient Chinese architecture is possible [9]. BIM parametric design is different from traditional collection form, as a new design method, based on a collection of BIM paying more attention to geometry and topology relationship between generation logic. Users can finish the work by modifying the program such as modify optimization deepen [10] upon a complete set of topological relations and generation logic system which can greatly reduce the workload of historical building repairs or restoration.

\section{"Time Value" and "Purpose Commemorative Value"__ The historical building maintenance management system based on 4D BIM}

The "Time Value" of Historical building is derived from the condensation in the historical buildings, so natural trail in historic buildings is the most direct embodiment for the appearance of the "Time Value". So the "Time Value" of the measures is to keep these changes triggered by nature. Adhering to the "Time Value" does not represent a narrow and negative, and prevent premature building too fast decay of measures must be taken. "Purpose Commemorate Value" belonging to the beginning of the construction is the memory of the historic buildings.

In simple terms, the $4 \mathrm{D}$ model is on the basis of the $3 \mathrm{D}$ model in the additional time factor, and the 4D model shows the formation process and changes in the form of dynamic 3D. Through a visual medium of the 4D model, the user can see the graphic simulation process of the objects change, so as to optimize and control the process of change. Late to the South Bank university building maintenance of the 4D model as an example, the researchers first calculate lighting material color in the maintenance plan of the building and natural environment under the influence. The successful use of the 4D model building maintenance of the South Bank College provides a new way for the research of building maintenance period and method [11]. Historical buildings as a purpose of memorial buildings exist forever and do not terminate the generation, the key also lies in the dynamic monitoring and maintenance. The 4D model nearly play an effect role in building maintenance, so can we apply this method further into the protection of historical buildings and set up a maintenance management system.

\section{Summary}

Seeing from the above analysis, BIM can effectively solve the difficulties that exist in the historical building protection, and extend the different value of historic buildings. We can put the BIM technology applied to the protection of the historic buildings as digital modernization of historic buildings to some extent. It is believed that BIM application type and will be more and applications will be more and more widely used with the specific application of the actual operational stronger and stronger. The modernization of historical building protection just around the corner!

\section{References:}

[1]Zhu Guangya and Yang Lixia. Historical building protection management confusion and thinking [J]. Architectural Journal, 2010(02): 18-22

[2] Li Hongyan. In reading of the Riegel historic buildings value theory. Architect, 2009(2): 41-46

[3]Xu Zongwu and Yang Changming. The reconsideration of historic building value . Architectural Journal, 2011(S2): 103-106.

[4]Zhao Jingxue, Jiang Li and Wang Huiyi. Researches on the application possibility of BIM technology in ancient buildings protection [Z]. Shang Hai, China: 20116 
[5]Zhu Lei. Practice analysis Early Ancient Chinese Wooden structure Building Information Modeling [D]. Tianjin University, 2012.

[6] Murphy M, Mcgovern E, Pavia S. Historic building information modelling (HBIM)[J]. Structural Survey, 2009, 27(4): 311-327

[7] Boeykens S, Himpe C, Martens B. A Case Study of Using BIM in Historical Reconstruction[J].

[8] Chen Yue. Parametric design of the Chinese ancient architecture[D]. Chongqing University, 2002

[9]Sun Weichao. A preliminary study on the design of ancient building information model system based on Architecture Revit[D]. Tianjin University, 2012

[10]Wang Xingyu. The parameterized design and the application of BIM technology[J]. Architecture Technique , 2011(6).

[11]Zhang Jianping, Cao Ming and Zhang Yang. Based on the IFC standard and engineering construction of 4D information model management system[J]. Engineering Mechanics, 2005(S1).

\section{Brief introduction of the authors:}

Li Yan (1977-), female, from Hengyang, Hunan Province; the associate professor of College of Civil Engineering and Architecture of Hainan University; the doctoral candidate of College of Economy and Management of Tianjin University; and mainly engaged in researches on historic buildings protection and development.

Contact Address: Hainan university institute of civil engineering, Haikou city, Hainan province.

Postcode: $570228 \quad$ Contact Number: 13976994448, 0898-36318668

Dong Kai(1978-), male, from Tianjin; the lecturer of College of Management and Economics of Tianjin Chengjian University; and mainly engaged in researches on information technology and image simulation.

Contact Address: Tianjin urban construction college of economics and management, Tianjin city, Hei Longjiang province.

Postcode: 300381

Contact Number: $13302076368,022-23085187$ 